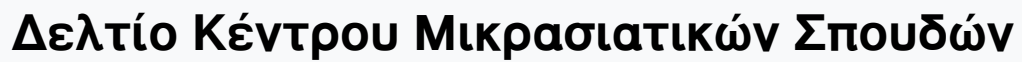

Tó 13 (1999)

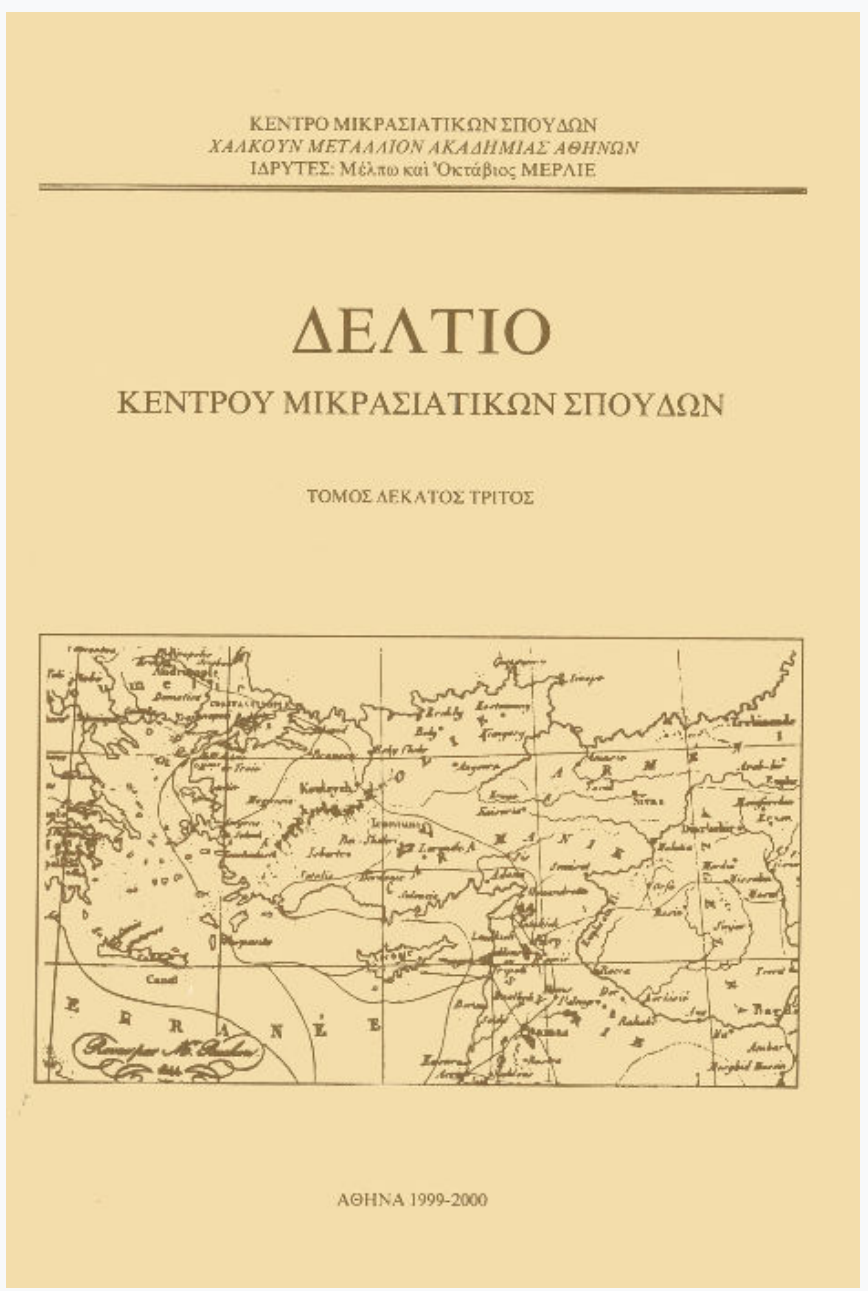

\section{Le pouvoir politique et les religions dans les Balkans}

\section{Asterios Argyriou}

doi: $10.12681 /$ deltiokms.153

\section{Copyright @ 2015, Asterios Argyriou}

\section{(c) (i) (2) (2)}

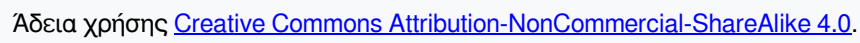

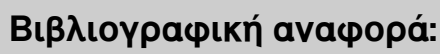

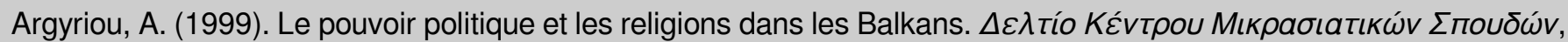
13, 363-366. https://doi.org/10.12681/deltiokms.153 


\section{ASTÉRIOS ARGYRIOU}

\section{LE POUVOIR POLITIQUE ET LES RELIGIONS DANS LES BALKANS}

Je me suis souvent posé la question de savoir si le précept évangélique «Rendez à César ce qui est à César et à Dieu ce qui est à Dieu» est un précept applicable dans notre région balkanique. En fait, pour me donner une réponse à cette question, je me dois d'abord savoir ce qui appartient à César et ce qui appartient à Dieu dans ce cas bien précis.

À une époque déjà très lointaine, le pouvoir politique byzantin, pour se soumettre les peuples slaves des Balkans, leur avait envoyé des missionnaires. Christianisés et munis d'une écriture, ceux-ci se créèrent, dans le sillage politique, religieux et culturel de l'Empire, une histoire et une culture nouvelles et se forgèrent une nouvelle conscience identitaire qui les poussera bientôt vers la création d'états, d'empires et d'Églises orthodoxes indépendants au détriment des Byzantins.

Puis sont venus les Turcs ottomans qui détruisirent l'empire chrétien d'Orient et soumirent à leur pouvoir la presque totalité des peuples orthodoxes des Balkans. Cette soumission avait un caractère foncièrement religieux. Les musulmans étaient les maitres, les citoyens de plein droit, tandis que les chrétiens se voyaient octroyer un statut de citoyens de seconde catégorie avec peu de droits et un nombre considérable d'obligations humiliantes et avilissantes. Il n'y avait qu'une seule échappatoire, adhérer à la religion des maîtres pour pouvoir accéder à la citoyenneté musulmane.

De longs siècles durant, la religion va donc jouer le rôle le plus déterminant dans le développement des mentalités et des cultures, dans la formation des 
consciences et des identités. La religion orthodoxe, véhiculée à travers une structure ecclésiastique que le pouvoir ottoman avait voulue ou tolérée, sera ce mortier qui cimentera très solidement l'asservissement commun et les souffrances multiples, matérielles et spirituelles, des peuples orthodoxes balkaniques. Dans un premier temps, une conscience très forte verra le jour et se développera, la conscience de former une nation cohérente et solidaire, la nation des orthodoxes face à la nation des musulmans et à celle des Latins, ou bien la nation des fidèles face à celle des infidèles et à celle des hérétiques. Et ce sera cette conscience, profondément religieuse et exempte de tout autre différenciation, qui sera la force motrice de leur devenir et de leur résistance à toutes les formes d'aliénation et de reniement. Les revendications aux droits à la différence ethnique, culturelle et linguistique n'y sont certes pas absentes, mais elles n'interfèrent que faiblement dans l'évolution générale de l'orthodoxie balkanique. Leur rôle sera prépondérant à partir de la fin du XVIIIe siècle, avec la pénétration dans le système éducatif orthodoxe des idées politiques des Lumières européennes et de la Révolution française.

Mais à ce moment également, moment déterminant pour le devenir des peuples balkaniques, la religion orthodoxe jouera son rôle primordial. Devenue orthodoxie nationale et parfois même nationaliste, elle insufflera, inspirera, portera dans ses bras, marquera de son sceau les revendications à l'indépendance et à l'identité nationale, culturelle, linguistique, territoriale etc. La création des états balkaniques nationaux allait de pair avec la création des Églises nationales, le création d'Églises nationales autocéphales ou de patriarcats nationaux. Si le patriarcat de Moscou avait été créé, en 1593, dans le but de sauvegarder l'intérêt commun à l'ensemble du monde orthodoxe asservi, les Églises balkaniques autocéphales ou patriarcales du XIXe siècle doivent leur création au seul critère de l'intérêt national. L'Église avait soutenu le peuple orthodoxe durant toute la période de son asservissement, avait souffert avec lui, l'avait protégé, guidé, nourri spirituellement et culturellement, l'avait encouragé dans ses luttes, combattu avec lui les armes à la main. Dès lors, comment pouvait-elle se désolidariser de son peuple ou être abandonnée par lui au moment ou celui-ci était sur le chemin de recouvrer son indépendance, sa dignité et son existence propre? Ainsi donc l'orthodoxie va devenir une orthodoxie nationale, elle sera partie prenante dans l'aventure de la formation des états indépendants, elle en sera l'une de leurs forces constitutives et constituantes. En effet, aucune histoire, aucune culture, aucune langue nationale balkanique ne saurait se soustraire à l'action sur elle de la religion, en premier lieu de la religion orthodoxe mais aussi 
de l'islam et du catholicisme pour certaines de nos régions. Il n'existe aucune frange de la vie, de la création et des aspirations des peuples et des états balkaniques qui ne porte profondément gravé sur son coeur et sur son âme le sceau de la religion. Ce ne sont donc pas seulement les édifices du culte, l'art sacré, l'histoire ecclésiastique de chacune de nos contrées qui portent sur eux cette empreinte, c'est notre être même dans sa totalité. Dans ce cas, comment pouvoir distinguer ce qui appartient à César et ce qui appartient à Dieu? Ainsi, je reviens au début de mon propos.

Mais je vais laisser à d'autres le domaine de César et m'appliquerai à faire quelques réflexions à propos du domaine de Dieu. Car il est évident qu'à Dieu revient en premier lieu la foi des hommes, la révélation sur laquelle cette foi est fondée, les principes qui commandent la pratique consciente et éclairée de cette foi dans le devenir historique et la vie quotidienne des peuples et des individus. Or la foi nour enseigne (la foi orthodoxe aussi bien que la foi musulmane ou catholique) que Dieu créa l'homme et le chargea de la mission d'être le vicaire, le gérant de sa création; que le Dieu Créateur envoie le soleil, la pluie et tous ses bienfaits à tous les hommes, aux bons comme aux méchants, à ses fidèles serviteurs comme aux rebelles à la révélation de sa volonté; qu'il désire le salut de tous les hommes; que l'épanouissement (le salut) de l'homme dans la totalité de sa personne a besoin d'une nourriture non seulement spirituelle mais aussi matérielle; que tout homme, qui que ce soit, le fidèle comme l'infidèle, a droit à la vie, au respect de la dignité et de l'intégrité de sa personne, le droit d'habiter la terre que Dieu a créée pour lui et sur laquelle il est établi, de jouir des bienfaits de son Créateur, d'avoir sa foi propre et la possibilité de la pratiquer librement et sans entraves, d'appartenir sans préjudices à la communauté ethnique, culturelle, linguistique et religieuse dans laquelle il est né et élevé ou bien qu'il se serait choisie.

Les religions, les Églises, les communautés religieuses et les individus qui se réclament de Dieu doivent, en premier lieu et avant tout, sauvegarder, défendre et appliquer précisement ces principes fondamentaux de leur foi religieuse; ils doivent savoir distinguer ce qui est à César et ce qui est à Dieu. Or à Dieu appartient l'homme dans toute sa dimension et quelle que soit son appartenance religieuse, ethnique, culturelle ou linguistique. C'est donc l'homme que les gens de la religion doivent rendre à Dieu. Et ils le rendront à Dieu, son Créateur, en défendant son droit à la vie, au respect de sa dignité personnelle. Plutôt que de se lier à César ou de s'identifier à lui, les hommes de Dieu doivent défendre et appliquer la volonté de Dieu. Une Église nationale ou une communauté 
religieuse particuliaire ne saurait prétendre appartenir à Dieu et agir en son nom, si elle se complaisait à la défense des droits des hommes de son Église ou de sa communauté uniquement, aussi légitime qu'elle puisse paraître parfois une telle attitude; elle ne saurait se réclamer du Dieu de l'amour, du Père de tous les hommes, si, au nom des droits légitimes d'une communauté déterminée, elle acceptait de se voir nier aux autres communautés et aux autres hommes ces mêmes droits. César appartient peut-être à un peuple, à une communauté, à un territoire, à une histoire particulière; Dieu est le Créateur et le Père commun de tous les hommes. Les religions et les Églises qui se réclament de ce Dieu doivent rendre à Dieu ce qui lui appartient, si elles ne veulent pas faillir à leur mission et à leur vocation, quoi que cela puisse leur coûter dans leurs rapports toujours difficiles et conflictuels avec César, le Prince de ce monde.

Si donc César se décide de porter atteinte à la vie ou à l'intégrité, au bien-être matériel ou spirituel d'un individu, d'une communauté, d'un peuple, parce qu'ils revendiquent une autre identité ethnique, linguistique, culturelle ou religieuse ou se réclament d'une autre idéologie, les hommes de religion doivent prendre la défense et chercher à adoucir les souffrances des enfants de Dieu atteints dans leur personne ou leurs biens à cause de leur différence, au nom justement de leur droit à la différence; prendre la défense de la misère contre l'opulence opprimante, des minorités faibles contre les majorités puissantes, du musulman, du chrétien, du Turc, du Grec, du Serbe, de l'Albanais, lorsque César l'opprime, le persécute, le prive de ses biens et de ses droits à cause de sa différence, quelle que soit celle-ci. La religion ne saurait en aucun cas être l'épée dans la main de César, servir d'alibi aux ambitions politiques d'un État, d'une communauté, d'un individu, devenir le substrat d'une idéologie quelconque. Une religion ou une Église ne saurait être considérée comme étant la religion ou l'Église d'un État que si elle est capable d'insuffler sur celui-ci le respect des droits inaliénables de tous les citoyens sans exception. Autrement elle devra entrer en conflit avec le pouvoir séculier, car elle aura choisi la partie du faible et de l'opprimé. À la religion, son Royaume n'est pas de ce monde, et lorsqu'elle s'y installe confortablement, c'est qu'elle a failli à sa mission et à sa vocation. 